\title{
CUNR scoring system for the prediction of lateral Iymph node metastasis in papillary thyroid carcinoma
}

\author{
Jianyong Lei ${ }^{1}$, Gengpeng Li $^{1}$, Zhihui Li ${ }^{1}$, RX Rong ${ }^{1}$ and Jingqiang Zhu ${ }^{1}$ \\ ${ }^{1}$ Thyroid and Parathyroid Surgery Center, West China Hospital of Sichuan University, Chengdu 610041, China \\ Correspondence to: Jingqiang Zhu, email: zjqdoctor@163.com \\ Keywords: papillary; thyroid carcinoma; lateral; lymph node; metastasis \\ Received: July 19, $2017 \quad$ Accepted: November 14, $2017 \quad$ Published: November 30, 2017 \\ Copyright: Lei et al. This is an open-access article distributed under the terms of the Creative Commons Attribution License 3.0 (CC \\ BY 3.0), which permits unrestricted use, distribution, and reproduction in any medium, provided the original author and source are \\ credited.
}

\section{ABSTRACT}

Objective: Our present study aimed to evaluate and compare the number and rate of central lymph node metastases (LNMs) for the prediction of lateral LNM (LLNM) in papillary thyroid carcinoma (PTC) and to develop a scoring system.

Results: Capsule invasion, tumor location in the upper portion of the thyroid, an ipsilateral central compartment LNM number $\geq 3$, and an ipsilateral central compartment LNM rate of $\geq 56 \%$ were identified as significant independent predictors of ipsilateral lateral LNM in PTC. The predictive ability of an ipsilateral central compartment LNM rate $\geq 56 \%$ (area under the curve $(A U C)=0.802$ ) was better than that of an ipsilateral central compartment LNM number $\geq 3$ ( $A U C=0.755$ ). The ROC curves identified the best index point (CUNR) to distinguish the presence or absence of ipsilateral LLNM as 11 , which has a high sensitivity $(0.860)$ and a low false-negative rate $(0.100,1$-Specificity). These findings were supported by the validation cohort.

Conclusions: Patients with a CUNR index point equal to or greater than 11 and ipsilateral lateral lymph node dissection should be considered for a diagnosis of LLNM.

Patients and Methods: A total of 1,281 PTC patients were included and divided into two groups: those with a presence of $\operatorname{LLNM}(n=222)$ and those with an absence of LLNM $(n=1059)$. Univariate and multivariate analyses were performed to detect the risk factors for LLNM, and receiver operating characteristic (ROC) curves were used to detect the best cutoff values of these predictors. Additionally, a scoring system for the odds ratio (OR) of independent factors was developed and validated in an independent cohort of PTC patients $(n=560)$.

\section{INTRODUCTION}

Papillary thyroid carcinoma (PTC) is the most common primary endocrine-related malignancy because it accounts for approximately $90 \%$ of all thyroid cancer cases [1]; currently, the incidence of PTC is rapidly increasing worldwide [2], particularly in Asian countries [3]. PTC is the most typical prolymphatic tumor because 20-90\% regional lymph node metastases (LNM) are usually present at diagnosis $[4,5]$. Additionally, accumulating evidence shows that lymph node metastases adversely affect survival, particularly in older patients with larger tumors and extrathyroidal extension [6]. Thus, complete resection of the primary tumor and metastatic lymph nodes is a prerequisite for subsequent thyroidstimulating hormone (TSH) suppression and iodine 131 therapies. LNM is often elusive and difficult to detect and diagnose with preoperative ultrasonography, particularly in the lateral neck compartment, which exhibits variable sensitivities that range from 37 to $84 \%$ [6-8].

Prophylactic central lymph node dissection has been recommended to improve the prognosis of these patients. In general, PTC metastasis initially occurs in the central compartment (VI) and then spreads to the lateral compartment of the neck [9]; in rare cases, lateral lymph node metastasis (LLNM) skips over the central neck compartment (skip metastasis) $[10,11]$. Previous studies have proven that the presence of central neck 
lymph node metastasis is valuable in the prediction of LLNM [9, 12-15]. Significant positive values for the ability of central lymph nodes to predict LLNM ranged from 2 to $5[14,16,17]$. However, until now, no study has reported the predictive significance of the central lymph node metastatic (LNM) ratio on LLNM, and no study has compared the number and efficiency of the central LNM ratio and number in the prediction of LLNM. The current study was designed to investigate the significance of the central LNM ratio in the prediction of LLNM and to compare the effectiveness of using the central metastatic lymph node number and ratio. In addition, we developed a scoring system based on the odds ratio (OR) of the risk factors to predict LLNM.

\section{RESULTS}

\section{Patient demographics and tumor characteristics}

The clinical and pathological characteristics of the 1,281 PTC patients enrolled in this study are shown in Table 1. In all, 343 (26.8\%) patients were men, and 939 $(73.3 \%)$ were women; the mean age was 40.5 years, and $439(34.3 \%)$ patients were older than 45 years. All the patients underwent conventional open surgery, and Hashimoto's thyroiditis (HT) was present in 336 (26.2\%) patients with PTC. Chronic disease, including hypertension and diabetes, among others, was present in $216(16.9 \%)$ patients with PTC. Most of the cases $(859,67.1 \%)$ showed unifocality, and 447 cases $(34.9 \%)$ exhibited histological capsule invasion. Additionally, only $33(2.6 \%)$ patients demonstrated distant metastases prior to surgery. The overall rate of LLNM was 222 among 1,281 patients $(17.3 \%)$.

\section{Associations between the clinicopathological characteristics and LLNM in patients with PTC}

Two hundred twenty-two patients (17.3\%) were demonstrated to have LLNM in the final histopathological examination, whereas 1,050 patients $(82.7 \%)$ demonstrated no evidence of LNM at least six months after surgery. As shown in Table 2 , nodular goiters ( $p=0.037$ ) or Graves's disease $(p=0.038)$, capsule invasion $(p<0.001)$, tumor location in the upper portion of the thyroid $(p=0.004)$, an ipsilateral central compartment LNM number $\geq 3$ $(p<0.001)$, and an ipsilateral central compartment LNM rate $\geq 56 \%(p<0.001)$ were significantly associated with a higher prevalence of LLNM in the univariate analysis. The cutoff values of the ipsilateral central compartment LNM number and rate were calculated from the ROC curve, as shown in Figure 1A and 1B. In the multivariate analysis shown in Table 3 , capsule invasion $(\mathrm{OR}=2.683$, $p<0.001)$, tumor location in the upper portion of the thyroid $(\mathrm{OR}=2.261, p=0.002)$, an ipsilateral central compartment LNM number $\geq 3(\mathrm{OR}=2.784, p=0.003)$, and an ipsilateral central compartment LNM rate $\geq 56 \%$ $(\mathrm{OR}=7.950, p<0.001)$ were significant independent predictors of ipsilateral LLNM in PTC. Additionally, Pearson's correlation analysis indicated that the ipsilateral and contralateral lateral compartment LNM numbers were linearly correlated with the ipsilateral and contralateral central compartment LNM numbers, respectively, as shown in Figure 2A and 2B $(r=0.440$ and $r=0.606$, all $p<0.001)$.

Univariate and multivariate analyses were performed to examine the risk factors for contralateral lateral compartment LNM and revealed that a contralateral central compartment $\mathrm{LNM} \geq 3$ (OR $=9.865,95 \% \mathrm{CI}$, 7.564-9.992, $p<0.001)$ and a contralateral central compartment $\mathrm{LNM}$ rate $\geq 69 \%(\mathrm{OR}=64.068,95 \% \mathrm{CI}$, 8.667-473.584, $p<0.001)$ were significant predictors of contralateral LNM.

\section{ROC curve}

ROC curves were generated, and area under the curve (AUC) calculations were performed for the regression models of the continuous variables, as shown in Figure 1A and 1B. The Youden index was used to estimate the appropriate critical point. The ROC curve analysis identified 2.5 as the appropriate critical point for the ipsilateral central LNM number, and the ipsilateral central LNM rate was 0.560. Therefore, we defined the ipsilateral central LNM high number as $\geq 3$, and the ipsilateral central LNM high rate as $\geq 56 \%$. Based on the ROC curve analysis, 2.5 is the appropriate critical point for the contralateral central LNM number, and the contralateral center LNM rate was 0.690. Thus, we defined the contralateral central LNM high number as $\geq 3$ and the contralateral central LNM high rate as $\geq 69 \%$, as shown in Figure 3A and 3B.

As shown in Figure 4A, a comparison of the four predictive factors for ipsilateral lateral compartment LNM revealed the following predictive factors: ipsilateral central $\mathrm{LNM}$ rate $\geq 56 \%$ (AUC $=0.802)$, an ipsilateral central LNM number $\geq 3(\mathrm{AUC}=0.755)$, capsule invasion $(\mathrm{AUC}=0.659)$, and a tumor location in the upper portion of the thyroid (AUC $=0.560$ ). Moreover, the predictive factors of contralateral lateral LNM were a contralateral central LNM rate $\geq 69 \%(\mathrm{AUC}=0.939)$ and a contralateral central LNM number $\geq 3(\mathrm{AUC}=0.866)$, as shown in Figure 4B.

The characteristics of ipsilateral lateral compartment LNM are shown in Table 4; single-level LNM was observed in 35 cases. The most common levels were Level IV $(71.2 \%)$ and III (70.3\%), followed by Level II (41.0\%) and Level V (33.3\%). Double-level (51.8\%) LLNM was the most common type, followed by triple-level (26.1\%), single-level (15.8\%) and quadruple-level (6.3\%) LLNM. 
Table 1: Demographics and tumor characteristics of patients with PTC $(n=1281)$

\begin{tabular}{|c|c|}
\hline Characteristics & Results \\
\hline Age at diagnosis (mean $\pm \mathrm{SD}$, years) & $40.5 \pm 13.6$ \\
\hline Gender $(\mathrm{M} / \mathrm{F})$ & $342 / 939$ \\
\hline Race (Han/others) & $1267 / 14$ \\
\hline $\operatorname{BMI}\left(\mathrm{kg} / \mathrm{m}^{2}\right)$ & $22.7 \pm 3.6$ \\
\hline Chronic disease (yes/no) & $216 / 1065$ \\
\hline Autoimmune thyroid disease (yes/no) & $946 / 336$ \\
\hline Graves' disease (yes/no) & $24 / 1257$ \\
\hline Nodular goiters (yes/no) & $600 / 681$ \\
\hline $\operatorname{NLR}($ mean $\pm \mathrm{SD})$ & $1.9 \pm 0.9$ \\
\hline $\operatorname{PLR}($ mean $\pm \mathrm{SD})$ & $108.3 \pm 51.0$ \\
\hline TSH level (mU/L, mean \pm SD) & $3.2 \pm 3.0$ \\
\hline FT4 level $(\mathrm{pmol} / \mathrm{L}$, mean $\pm \mathrm{SD})$ & $17.4 \pm 5.6$ \\
\hline FT3 level $(\mathrm{pmol} / \mathrm{L}$, mean $\pm \mathrm{SD})$ & $4.9 \pm 0.7$ \\
\hline Multifocality (yes/no) & $211 / 1070$ \\
\hline Bilaterality (yes/no) & $115 / 1166$ \\
\hline Capsule invasion (yes/no) & $447 / 834$ \\
\hline Extrathyroid extension (yes/no) & $64 / 1217$ \\
\hline Total tumor size (mean $\pm \mathrm{SD}, \mathrm{mm})$ & $14.9 \pm 10.5$ \\
\hline Largest tumor size (mean $\pm \mathrm{SD}, \mathrm{mm})$ & $14.0 \pm 9.8$ \\
\hline Primary tumor location (Upper/Middle/Lower) & $522 / 397 / 362$ \\
\hline Tumor extension $(\mathrm{T} 1 / \mathrm{T} 2 / \mathrm{T} 3 / \mathrm{T} 4)$ & $448 / 114 / 539 / 180$ \\
\hline
\end{tabular}

Chronic disease: hypertension, diabetes and others;

SD: standard deviation; BMI: body mass index; NLR: neutrophil-to-lymphocyte ratio; PLR: platelet-to-lymphocyte ratio; TSH: thyroid stimulating hormone; FT3: free triiodothyronine; FT4: free thyroxine.

\section{Index points for the distinction of the presence or absence of LNM}

According to the multiple logistic regression analysis, four risk factors and two risk factors were statistically significantly associated with ipsilateral lateral compartment LNM and contralateral lateral compartment LNM, respectively, and the estimate and cutoff points for each characteristic are shown in Table 5. The sum of the points (CUNR) was evaluated for its ability to distinguish between the presence and absence of lateral LNM. As determined using the ROC curves, the best point with high sensitivity $(0.860)$ and a low false-negative rate (0.100, 1-Specificity) was 11 , and the AUC was 0.910 , as shown in Figure 5A. However, the calculated index points for contralateral lateral compartment LNM were 0, 10, 64, and 74, and the ROC curve is shown in Figure 5B; however, the calculated AUC is only 0.696, which is markedly lower than that for a contralateral central compartment $\mathrm{LNM}$ number $\geq 3(\mathrm{AUC}=0.866)$ and that for a contralateral central compartment LNM rate $\geq 69 \%$ $(\mathrm{AUC}=0.939)$.

\section{Independent validation of our scoring system}

Our scoring system for the prediction of lateral LNM was validated by an independent cohort of PTC patients at our center $(n=560)$, and the results revealed that our scoring system performed equally well. The AUC of the CUNR scoring for the prediction of ipsilateral lateral LNM was 0.848 in the validated cohort (as shown in Figure 7B), and this value was comparable with that obtained with the training cohort (as shown in Figure 7A, $\mathrm{AUC}=0.896 ; p$ value $>0.10$ ).

\section{Long-term tumor-free survival}

Eight patients with distant metastasis were excluded from this analysis, 20 patients $(9.0 \%)$ with preoperative LLNM were diagnosed with tumor recurrence or metastasis, and $57(5.5 \%)$ patients did not present with preoperative LLNM at least six months before surgery. Additionally, the tumor-free survival in the group without preoperative LLNM was significantly less than that of the group with preoperative lateral LNM (as shown in Figure $6, p=0.028$ ). For the 77 patients with recurrence or metastasis, the most common site was the cervical lymph nodes, followed by the lung, bone and liver.

\section{DISCUSSION}

PTC is one type of thyroid carcinoma that originates from follicular cells and that has a favorable long-term outcome. However, PTC frequently metastasizes to the cervical lymph nodes, and LNM is one of the significant predictive factors of a poor outcome, with high rates of 
Table 2: Univariate analysis of LLNM in patients with PTC

\begin{tabular}{|c|c|c|c|}
\hline \multirow[b]{2}{*}{ Variable } & \multicolumn{2}{|c|}{ Ipsilateral } & \multirow[b]{2}{*}{$p$ value } \\
\hline & $\begin{array}{l}\text { Presence of LLNM } \\
\quad(n=222)\end{array}$ & $\begin{array}{l}\text { Absence of LLNM } \\
\quad(n=1059)\end{array}$ & \\
\hline Age $(\leq 45 />45$ years $)$ & $150 / 72$ & $692 / 367$ & 0.526 \\
\hline Sex (male/female) & $59 / 163$ & $282 / 777$ & 0.987 \\
\hline Race (Han/other) & $221 / 1$ & $1046 / 13$ & 0.312 \\
\hline BMI $\left(<24 / \geq 24 \mathrm{~kg} / \mathrm{m}^{2}\right)$ & $149 / 73$ & $714 / 345$ & 0.930 \\
\hline Chronic disease (yes/no) & 29/193 & $187 / 872$ & 0.097 \\
\hline HD (yes/no) & $164 / 58$ & $782 / 277$ & 0.836 \\
\hline Graves’ disease (yes/no) & $8 / 214$ & $16 / 1043$ & $0.037^{*}$ \\
\hline Nodular goiters (yes/no) & $118 / 104$ & $482 / 577$ & $0.038^{*}$ \\
\hline $\operatorname{NLR}(\leq 2 />2)$ & $136 / 86$ & $694 / 365$ & 0.226 \\
\hline $\operatorname{PLR}(\leq 200 />200)$ & $215 / 7$ & $1007 / 52$ & 0.256 \\
\hline TSH level $(\leq 4.2 />4.2 \mathrm{mU} / \mathrm{L})$ & $186 / 36$ & $848 / 211$ & 0.252 \\
\hline Multifocality (yes/no) & $37 / 185$ & $885 / 174$ & 0.931 \\
\hline Capsule invasion (yes/no) & $136 / 86$ & $311 / 748$ & $<0.001^{*}$ \\
\hline Extrathyroid extension (yes/no) & $6 / 216$ & $58 / 1001$ & 0.085 \\
\hline Total tumor size $(\leq 10 \mathrm{~mm},>10 \mathrm{~mm})$ & $87 / 135$ & $487 / 572$ & 0.064 \\
\hline Primary tumor size $(\leq 10 \mathrm{~mm},>10 \mathrm{~mm})$ & $99 / 123$ & $523 / 364$ & 0.177 \\
\hline Tumor extension (T1-T2/T3-T4) & $87 / 135$ & $475 / 584$ & 0.122 \\
\hline Primary tumor location (upper/middle or lower) & $113 / 109$ & $413 / 646$ & $0.004^{*}$ \\
\hline Ipsilateral central compartment LNM number $(<3 / \geq 3)$ & 23/199 & $649 / 410$ & $<0.001^{*}$ \\
\hline Ipsilateral central compartment LNM rate $(<56 \% / \geq 56 \%)$ & $37 / 186$ & $811 / 248$ & $<0.001^{*}$ \\
\hline Contralateral central compartment LNM $(<3 / \geq 3)$ & $113 / 109$ & $798 / 261$ & $<0.001^{*}$ \\
\hline Contralateral central compartment LNM rate $(<69 \% / \geq 69 \%)$ & $158 / 64$ & $970 / 89$ & $<0.001^{*}$ \\
\hline
\end{tabular}

LLNM: lateral lymph node metastasis; BMI: body mass index; HD: Hashimoto thyroiditis; NLR: neutrophil-to-lymphocyte ratio; PLR: platelet-to-lymphocyte ratio; TSH: thyroid stimulating hormone; LNM: lymph node metastasis
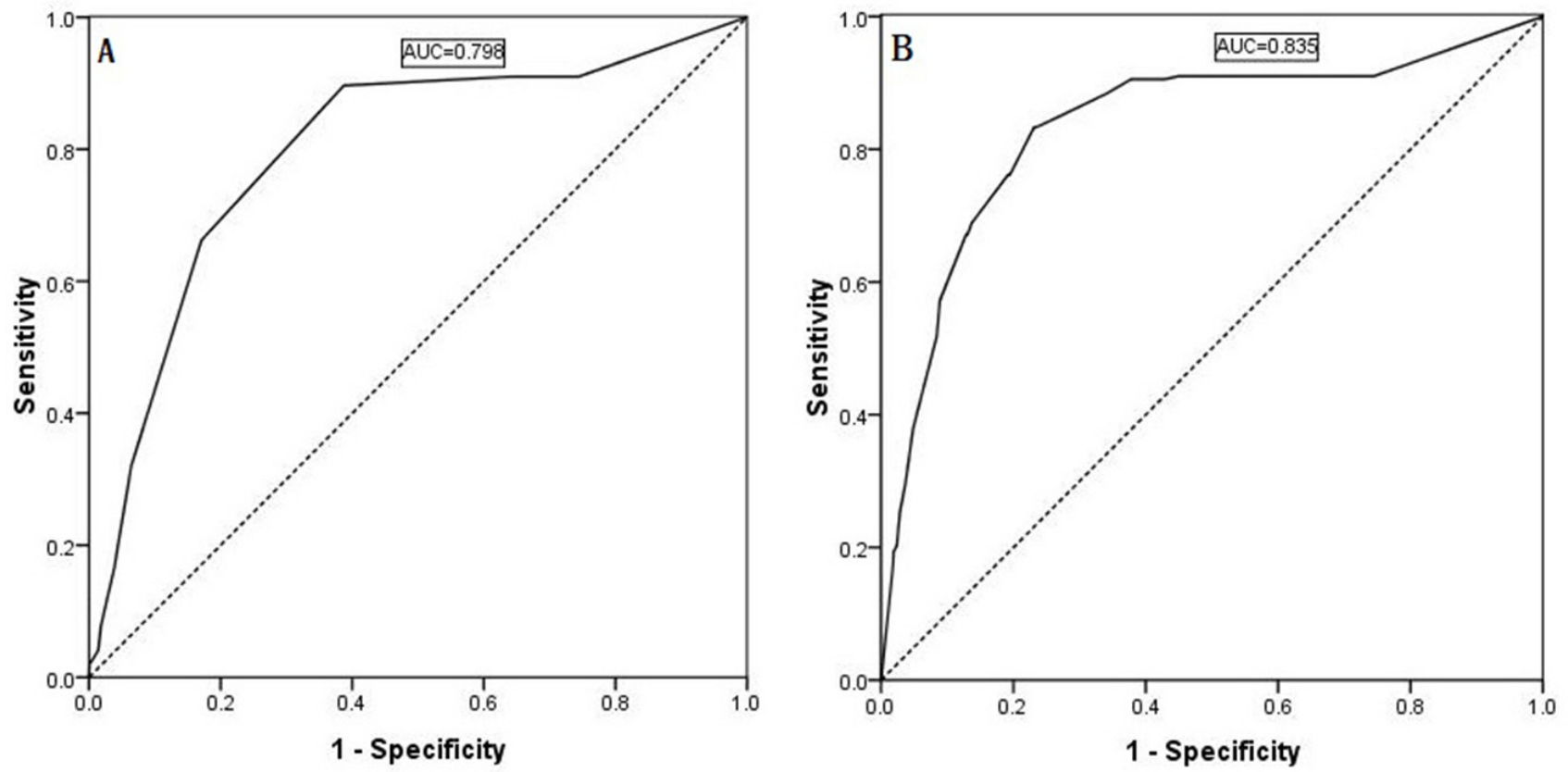

Figure 1: Predictability of ipsilateral lateral LNM shown by an ROC curve for the ipsilateral central compartment LNM number (A, AUC: 0.798 ) and the ipsilateral central compartment LNM rate (B, AUC: 0.835). 
Table 3: Multivariate analyses of factors contributing to LLNM in PTC

\begin{tabular}{|c|c|c|c|}
\hline Variables & Odds ratio & $95 \%$ CI & $p$ value \\
\hline \multicolumn{4}{|l|}{ Ipsilateral lateral compartment LNM risk factors } \\
\hline Capsule invasion (yes/no) & 2.683 & $1.895-3.798$ & $<0.001^{*}$ \\
\hline Primary Tumor location (upper/middle or lower) & 2.261 & $1.348-3.792$ & $0.002^{*}$ \\
\hline Ipsilateral central compartment LNM number $(\geq 3 /<3)$ & 2.784 & $1.418-5.464$ & $0.003^{*}$ \\
\hline Ipsilateral central compartment LNM rate $(\geq 56 \% /<56 \%)$ & 7.950 & $4.495-14.061$ & $<0.001^{*}$ \\
\hline \multicolumn{4}{|l|}{ Contralateral lateral compartment LNM risk factors } \\
\hline Contralateral central compartment LNM number $(\geq 3 /<3)$ & 9.865 & $7.564-9.992$ & $<0.001^{*}$ \\
\hline Contralateral central compartment LNM rate $(\geq 69 \% /<69 \%)$ & 64.068 & $8.667-473.584$ & $<0.001^{*}$ \\
\hline
\end{tabular}

LNM: lymph node metastasis; LLNM: lateral lymph node metastasis.

recurrence, distant metastasis and the requirement for more aggressive management when LNM is suspected [18]; thus, we focused on LNM of PTC. Unfortunately, the status of LNM is not obvious, and the way in which the status is determined is inadequate. Due to its high specificity and positive predictive value, ultrasonography is an important tool for the detection of metastatic nodes [19]; however, ultrasonography has limitations, including variable sensitivities (37-84\%) and difficulty in evaluating LNM $[7,8]$. As shown in recent studies, routine unilateral central lymph node dissection does not increase the risk of complications but might help determine the central lymph node status and could provide more accurate cancer staging information $[20,21]$. Thus, prophylactic central, but not prophylactic lateral, LND has been recommended for patients with PTC, and other clinicopathological predictive factors of LLNM might improve the selection of patients for lateral neck dissection.

Our hypothesis was that the number or rate of positive central lymph nodes is valuable in the prediction of the presence of LLNM. In general, cervical lymph node metastasis initially occurs in the central compartment

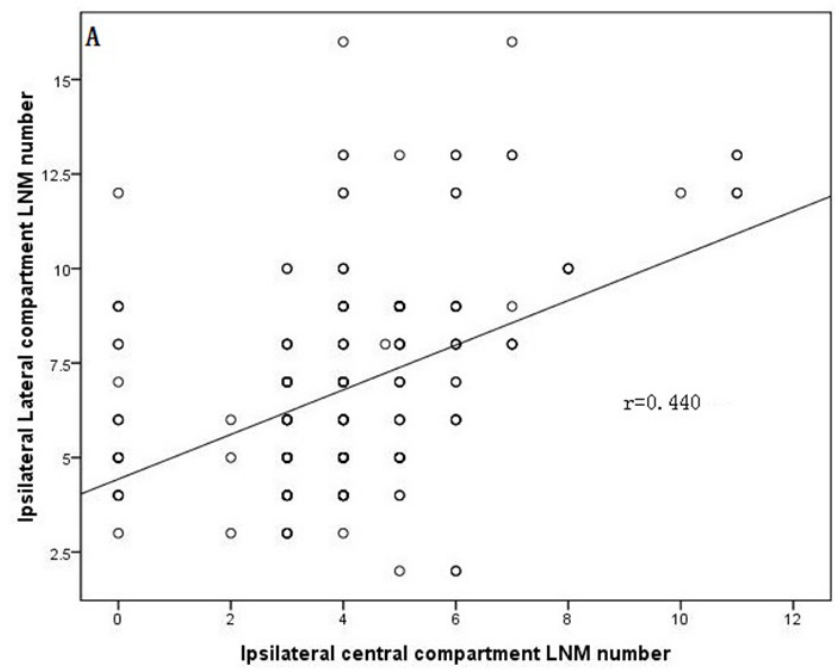

lymph nodes and subsequently in the lateral neck compartment [9], whereas "skip metastasis" occurs in very few patients with PTC $[22,23]$. Although this study is not the first to evaluate the ability of the number or rate of central LNM to predict LLNM $[6,15]$, to our knowledge, our study is the first to compare the ability of both the number and rate of central LNM for the prediction of LLNM; most importantly, we established index points based on the risk factors to distinguish patients with or without LNM. Moreover, we validated our CUNR scoring index independently in a large number of PTC patients. Our CUNR scoring system was valuable and feasible: first, unilateral central LND is routinely performed; and second, the frozen sections of the specimens dissected from the central compartment are analyzed in less than 30 minutes to determine the number and rate of central LNM [24].

As shown in previous studies, central lymph node metastasis is an important independent factor for LLNM, and most of these studies assessed the ability of the number of central LNMs to predict LLNM; however, the cutoff values vary. Machens et al [16] reported that the rates of LLNM increased from $45-69 \%$ to $100 \%$ in

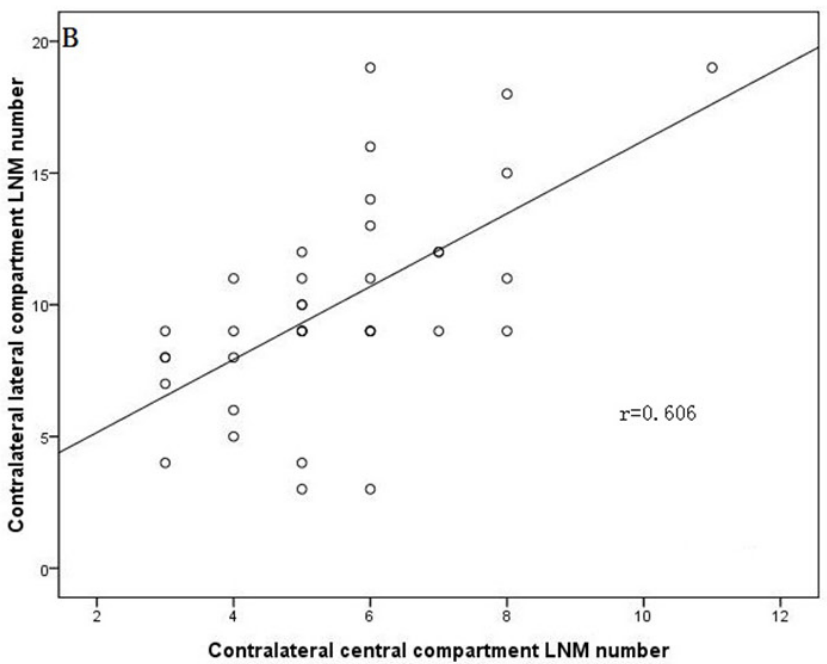

Figure 2: Ipsilateral and contralateral lateral compartment LNM numbers were linearly correlated with the ipsilateral $(\mathbf{A}, r=0.440)$ and contralateral central compartment LNM numbers $(\mathbf{B}, r=0.606)$. 
the ipsilateral neck and from $0-33 \%$ to $60-71 \%$ in the contralateral neck when patients had more than five central lymph nodes that were positive for metastases. Zeng et al. [14] and Xiao et al [17] reported that the presence of at least two positive central lymph nodes was an independent predictive factor for LLNM and should be valuable in the prediction of LLNM. Lee et al. [18] indicated that further treatment of the lateral compartment should be considered if the number of positive central lymph nodes is greater than two $(\geq 3)$, which is a finding that was consistent with our results. However, our cutoff value demonstrated greater predictive ability. First, our

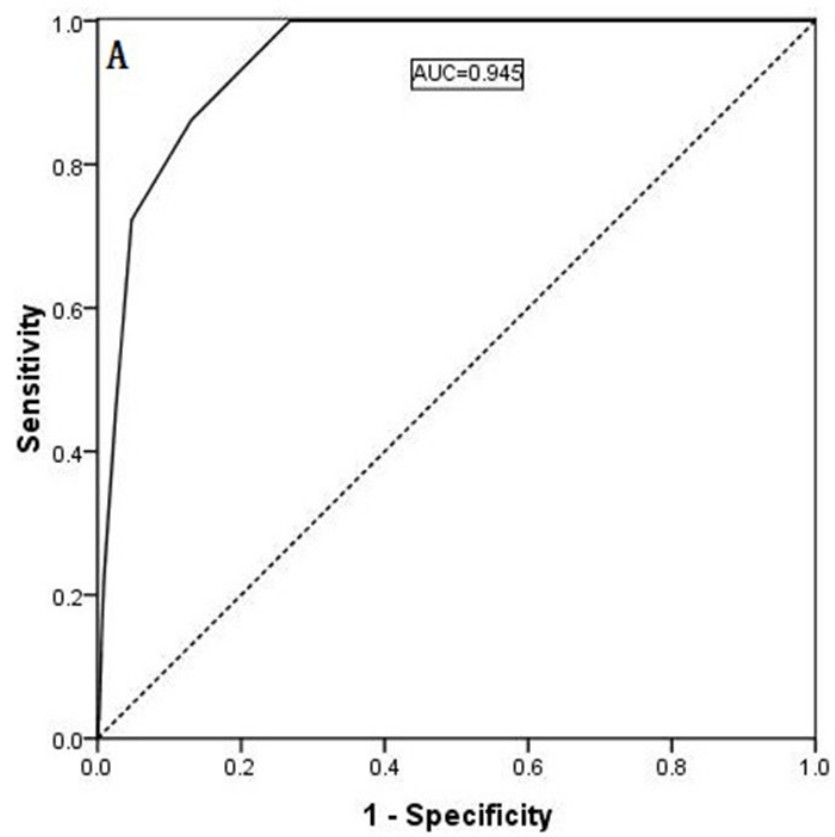

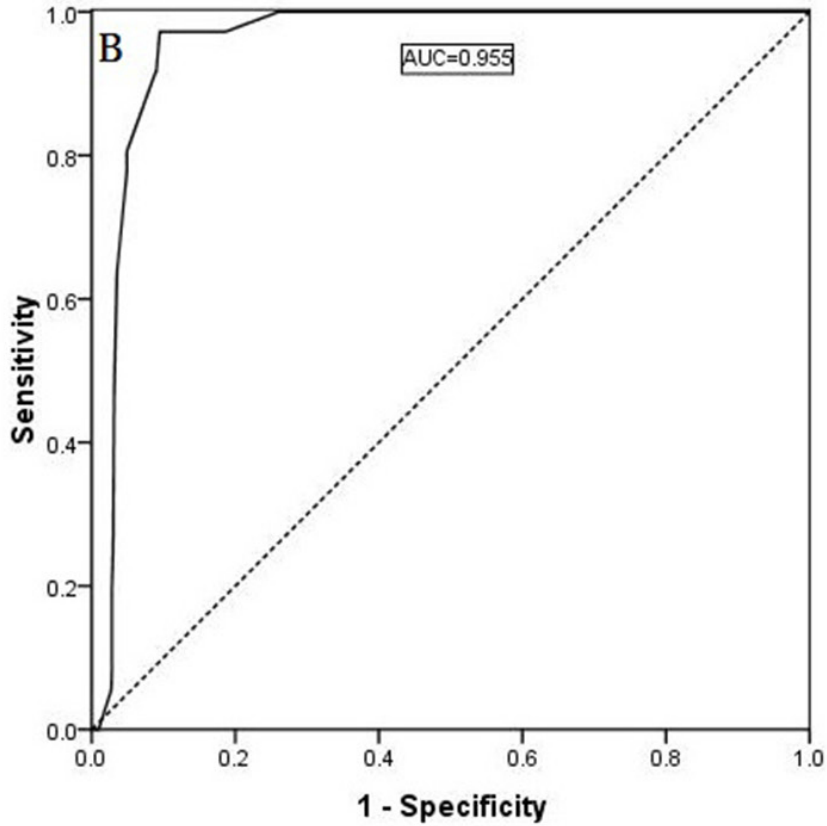

Figure 3: Predictability of contralateral lateral LNM shown by an ROC curve for the contralateral central compartment LNM number (A, AUC: 0.945) and the ipsilateral central compartment LNM rate (B, AUC: 0.955).
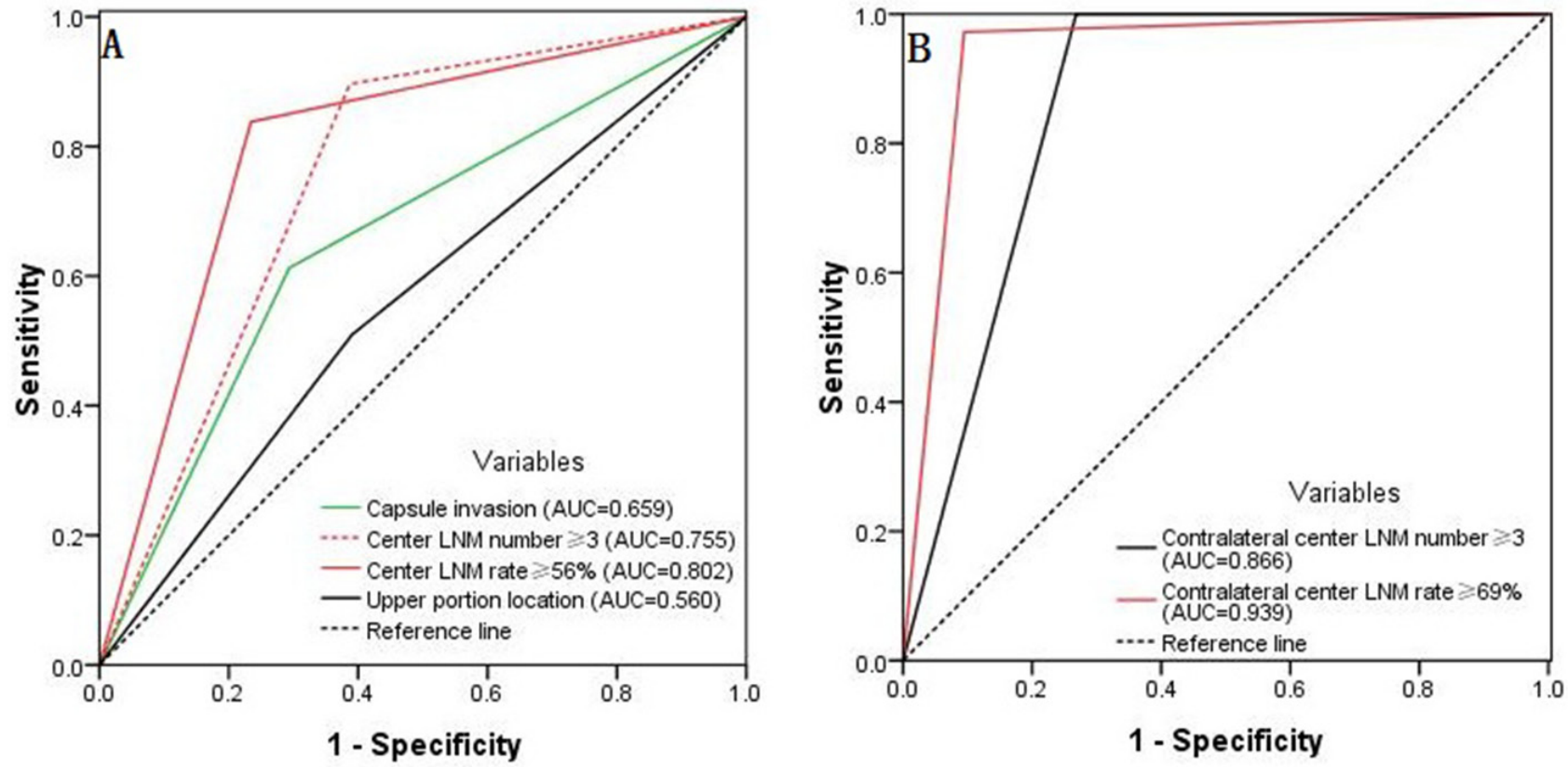

Figure 4: (A) The predictive factors for ipsilateral lateral compartment LNM were an ipsilateral central LNM rate $\geq 56 \%$, an ipsilateral central LNM number $\geq 3$, capsule invasion, and a tumor location in the upper portion of the thyroid. (B) The predictive factors for contralateral lateral LNM were a contralateral central LNM rate $\geq 69 \%$ and a contralateral central LNM number $\geq 3$. 
Distribution (II-V)

Single level (II/III/IV)

Lateral neck LN metastasis cases

Level II

Level III

Level IV

Level V

Lateral compartment single-level metastasis

Lateral compartment double-level metastasis

Lateral compartment triple-level metastasis

Lateral compartment four-level metastasis
Number of patients

$10 / 4 / 17 / 4$

91

156

158

74

35

115

58

14

Table 5: CUNR Index points for ipsilateral LLNM

\begin{tabular}{lccc}
\hline Variables & CUNR & Odds ratio & Index points \\
\hline Ipsilateral lateral compartment LNM risk factors & & & $16 / 0$ \\
Capsule invasion (yes/no) & $\mathrm{C}$ & 2.683 & $3 / 0$ \\
Primary tumor location(upper/middle or lower) & $\mathrm{U}$ & 2.261 & $2 / 0$ \\
Ipsilateral central compartment LNM number $(\geq 3 /<3)$ & $\mathrm{N}$ & 2.784 & $3 / 0$ \\
Ipsilateral central compartment LNM rate $(\geq 56 \% /<56 \%)$ & $\mathrm{R}$ & 7.950 & $8 / 0$ \\
Contralateral lateral compartment LNM risk factors & & & $74 / 0$ \\
Contralateral central compartment LNM $(\geq 3 /<3)$ & & 9.865 & $10 / 0$ \\
Contralateral central compartment LNM rate $(\geq 69 \% /<69 \%)$ & & 64.068 & $64 / 0$ \\
\hline
\end{tabular}

total number of patients with PTC was larger than that in previous studies; second, the cutoff value was calculated from the ROC curve and was verified through univariate and multivariate analyses. Finally, the combination of all the predictive factors was used to establish a scoring system to predict ipsilateral LLNM.
Only a few studies have evaluated the central metastatic lymph node ratio. Zhu et al. [15] found that the high number for central LNM was $\geq 4$ and that the high rate for central LNM was $\geq 40 \%$ for the prediction of LLNM. In our study, the cutoff value for the central LNM rate for the prediction of ipsilateral LLNM was
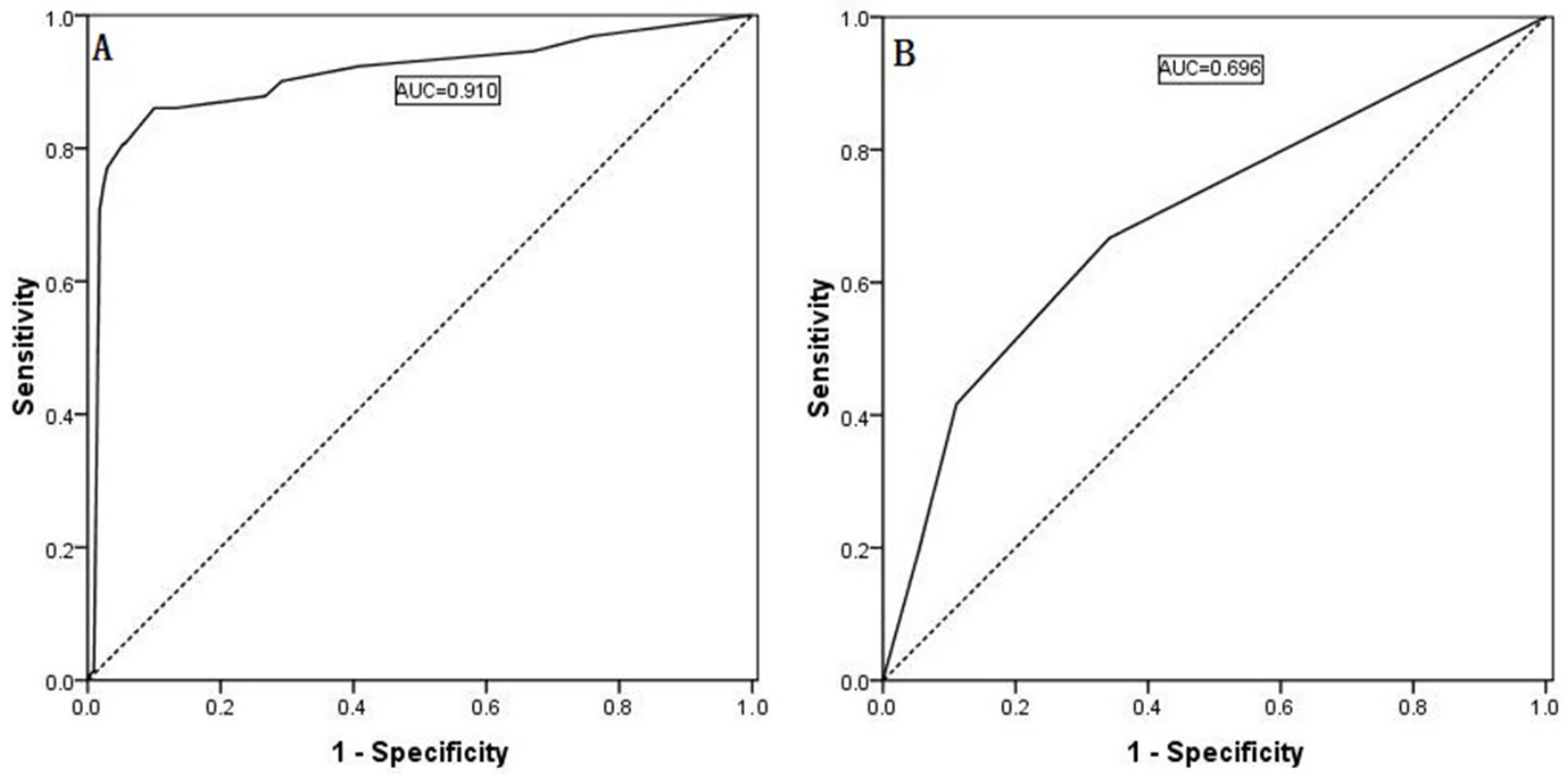

Figure 5: (A) Predictability of ipsilateral lateral LNM as shown by an ROC curve based on the scoring system. The best point with the highest sensitivity and the lowest false-negative rate was 11; the AUC was 0.910. (B) The calculated index points for contralateral lateral compartment LNM were 0, 10, 64, and 74. 


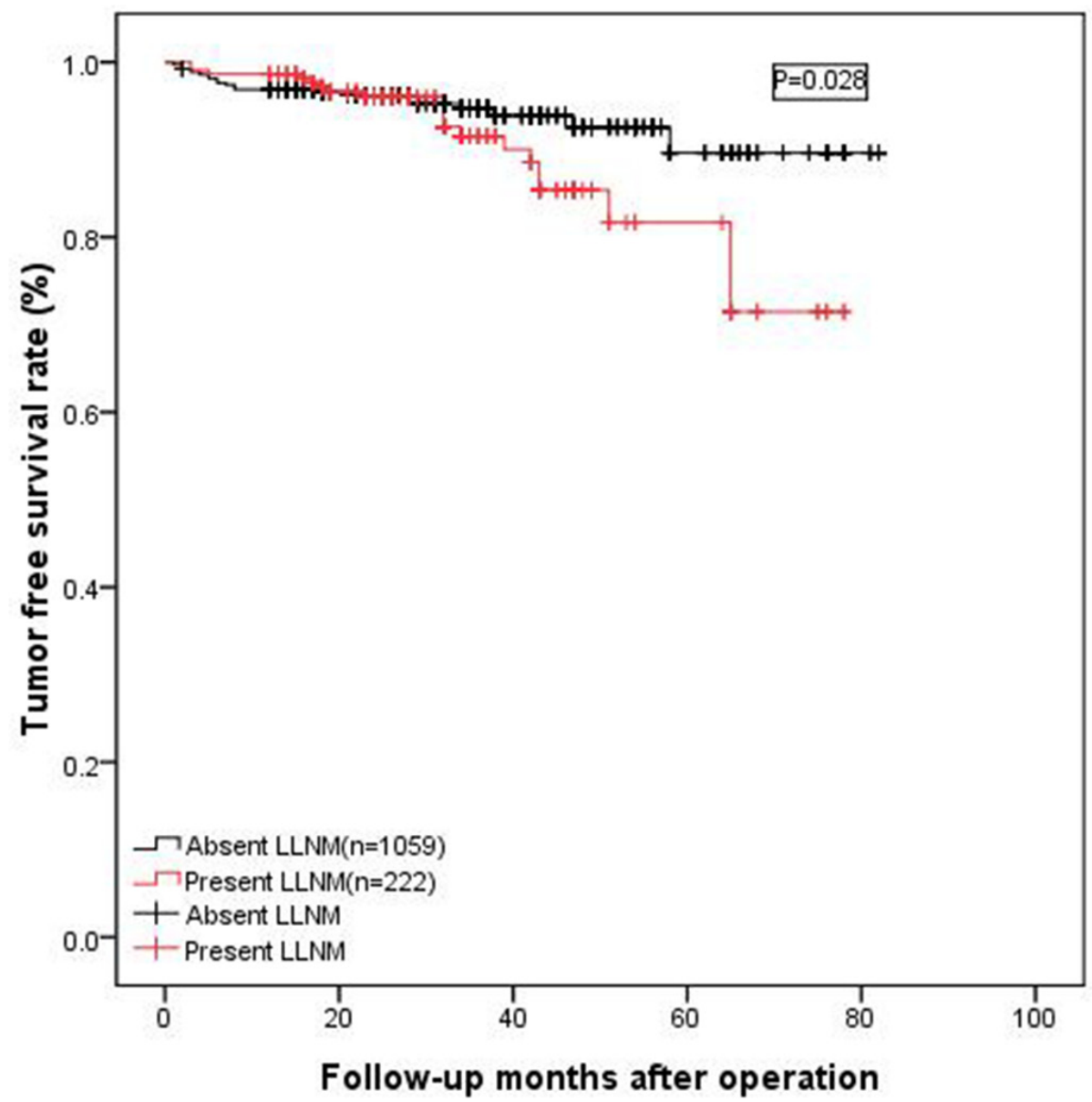

Figure 6: The tumor-free survival rate of patients without preoperative LLNM was significantly lower than that of patients with preoperative lateral LNM $(p=0.028)$.
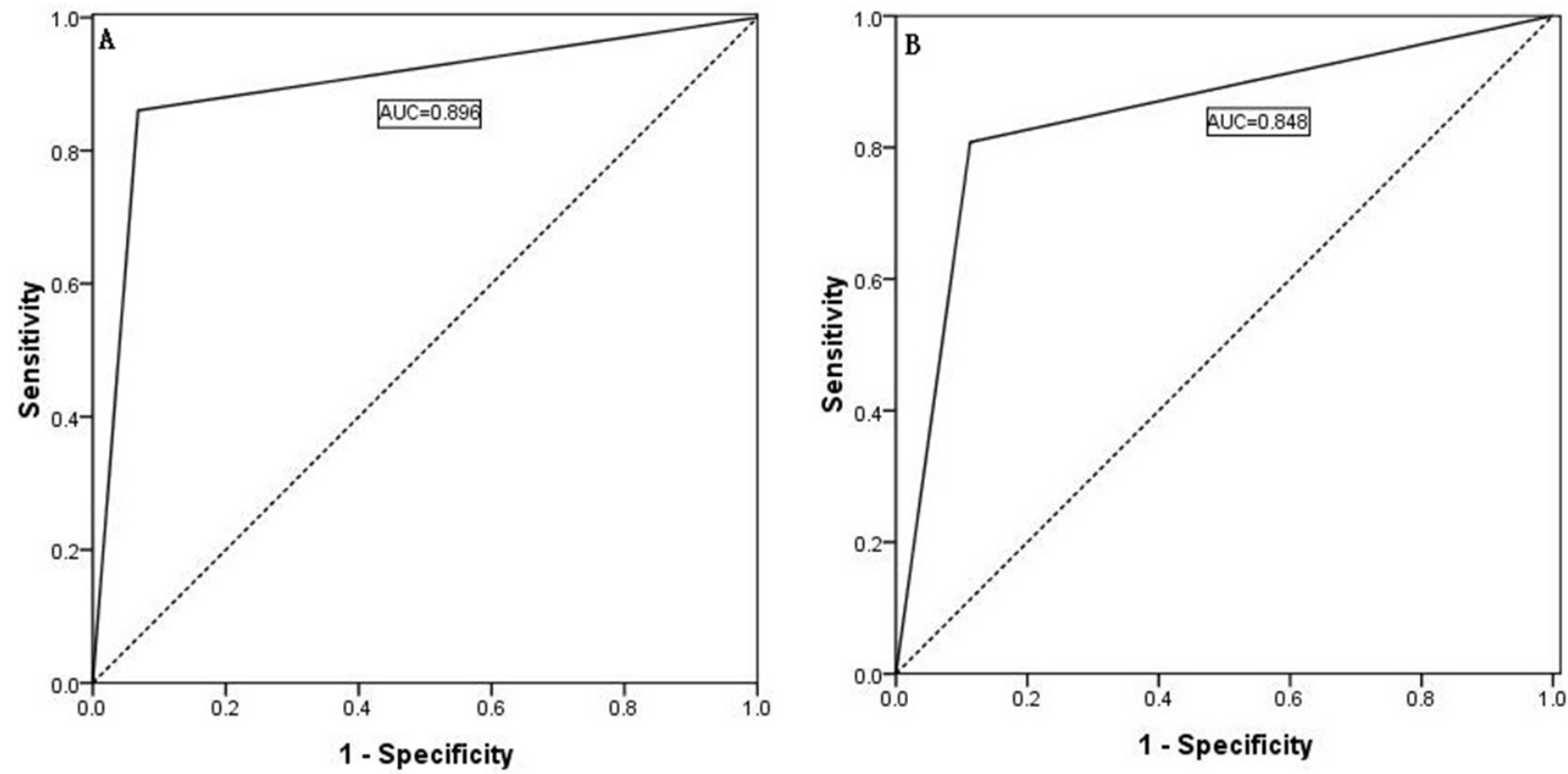

Figure 7: (A) Predictivity of the CUNR score index for ipsilateral lateral compartment LNM in the training cohort; (B) Predictivity of the CUNR score index in the validation cohort. 
$56 \%$, which is higher than the value obtained in the study by Zhu et al. Moreover, univariate and multivariate analyses indicated that the number and rate of contralateral central compartment LNM were also risk factors for contralateral lateral compartment LNM; the OR of the rate of contralateral central compartment LNM was 64.068, which is significantly higher than the OR of the number of contralateral central compartment LNM (9.865). Thus, compared with the number of central LNM, the rate of central LNM was more valuable for the prediction of LLNM, including ipsilateral and contralateral LLNM.

The association between HT and LNM remains controversial. Patients with HT may have less extrathyroidal extension, less extranodal spread, a lower rate of central and lateral LNM, a lower TNM score [15], better prognosis, and a lower recurrence rate [25], which has led to the hypothesis that the lymphocytic response limits tumor growth and metastases [26]. However, in patients with PTC, LLNM and HT were more frequently observed in another study [27]. Capsule invasion proved to be an independent factor for the presence of LNM. In our present study, the univariate and multivariate analyses indicated that an ipsilateral central compartment LNM rate $\geq 56 \%$ (OR $=7.950)$, an ipsilateral central compartment LNM number $\geq 3 \quad(\mathrm{OR}=2.784)$, capsule invasion $(\mathrm{OR}=2.683)$ and a location of the primary tumor in the upper thyroid $(\mathrm{OR}=2.261)$ were independent predictive factors of LLNM. Furthermore, an ipsilateral central compartment LNM rate $\geq 56 \%$, which had the highest OR value, was a key factor in predicting ipsilateral lateral LNM. Based on the OR value, we established a scoring system and determined that the best cutoff value for the prediction of LLNM with high sensitivity (0.860) and specificity (0.900) was 11 (AUC $=0.910)$.

This study had several limitations, such as the inevitable inherent features of a retrospective and nonrandomized cohort study. Because LNM was evaluated only for therapeutic purposes at our center, residual subclinical LLNM might have been present; however, prophylactic LNM is not generally recommended by the ATA or ETA guidelines, and thus, this limitation is inevitable. Moreover, our predictive index point cannot replace ultrasonography, FNA or thyroglobulin (Tg) levels in the diagnosis of lateral LNM but might still be helpful. Therefore, larger randomized multicenter studies are needed to confirm our results.

The CUNR scoring system for the risk factors that predict LLNM might be another approach that can be used for the diagnosis of LLNM and for the guidance of lateral LND. Patients with index points $\geq 11$ should be considered ipsilateral LLNM-positive; when the rate of contralateral central compartment LNM is $\geq 69 \%$, contralateral LLNM should be considered. However, the CUNR scoring system should be used only as an adjunct to ultrasonography and pathology.

\section{PATIENTS AND METHODS}

The institutional review board of West China Hospital approved this retrospective observational study, and the patients provided approval and written informed consent prior to surgery; further patient approval or informed consent was not required for our review of the patients' medical records. From December 2008 to December 2015, 3,385 consecutive patients who underwent surgery for PTC at our center were included in the present study. The inclusion criteria for the present study were as follows: patients with PTC who underwent total or near-total thyroidectomy and patients who underwent bi-central lymph node dissection (LND). The exclusion criteria were as follows: patients with other types of thyroid malignancies, such as follicular or medullary carcinoma; patients with a history or file record of thyroid or neck surgery for nonthyroid head and neck carcinomas; and patients with bilateral lobe PTC. Based on these criteria, 1,281 patients with PTC were included in the present study.

Ultrasonography and neck-enhanced computed tomography (CT) or magnetic resonance imaging (MRI) were routinely performed on all patients prior to surgery, and the imaging results were co-evaluated by a radiologist and a surgeon with at least 10 years of experience with overall subject evaluation. In cases where PTC was suspected (presence of central necrosis or cystic changes, dense cortical enhancement, nonparallel shape, hypoechogenicity, irregular margins, or microcalcification), fine-needle aspiration biopsy (FNAB) and cytological BRAF ${ }^{\mathrm{V} 600 \mathrm{E}}$ mutation analyses were performed to confirm the diagnosis. All lateral neck lymph node compartment dissections were performed in patients with PTC who were confirmed to have clinical evidence of positive lateral neck nodes (no prophylactic lateral lymph node dissection) via FNAB or FNA thyroglobulin assessment; in other cases, an intraoperative frozen biopsy demonstrated the presence of LLNM. Level II, III, IV, and V LLNM cases were routinely dissected. Prophylactic bilateral central neck dissection (bi-CND) was performed on all of the patients with PTC and proven LLNM at our center, and prophylactic contralateral central compartment LN dissection was performed if prelaryngeal or pretracheal lymph node (LN) metastasis was diagnosed through the assessment of intraoperative frozen sections [28]. The central lymph node compartment was defined as the region superior to the thyroid cartilage notch, lateral to the carotid sheaths, posterior to the prevertebral fascia, including VI A (superficial right recurrent laryngeal nerve) and VI B (posterior of the right recurrent laryngeal nerve), inferior to the innominate vein, and inboard to the tracheal sidewall. All surgical procedures were performed by one of three experienced surgeons with at least 15 years of thyroid surgery experience. The histopathological evaluation of the thyroid specimens 
and lymph node metastases was performed independently by two experienced pathologists with at least 10 years of experience in the diagnosis of over 500 cases of PTC. We defined capsule invasion as invasion of the thyroid capsule without organ or soft tissue invasion, as observed on microscopy. Moreover, we defined extrathyroid extension as the presence of soft tissue/organ invasion, as shown on microscopy [29].

Postsurgical radioiodine ablation was applied according to the postoperative pathological findings, and postoperative TSH suppression therapy was based on the ATA guidelines [30]. We defined the central compartment as the region located cranially to both superior thyroid arteries and to the pyramidal lobe, caudally to the innominate vein, laterally to the carotid sheaths and dorsally to the prevertebral fascia [31]. We distinguished recurrence by the presence of residual tumor at a follow-up duration of more than six months after initial surgery [32].

To avoid overoptimistic results due to model development and evaluation using the same dataset, the predictive performance of the scoring system was assessed in an independent validation cohort.

All data were managed and analyzed using SPSS software (version 17; SPSS, Inc., Chicago, IL, USA). Continuous and categorical data are expressed as the means \pm SD and rates, respectively, and the differences were compared and analyzed using the Mann-Whitney $U$-test and chi-squared test or Fisher's exact test (twotailed), if necessary. Univariate analyses were used to test the association with LLNM. Multivariate logistic regression was performed on all variables that were significant in the univariate analysis. ORs and 95\% relative confidence intervals (CIs) were calculated to determine the relevance of all potential predictors. Receiver operating characteristic (ROC) curves were used to determine the optimal cutoff values for the rate and number of central lymph node metastases (CLM) in the prediction of LLNM. According to multiple logistic regression analyses, features that were independent factors were assigned different points based on the OR to develop a scoring system, and the best point with a high sensitivity and low false-negative rate (1-Specificity) was then identified. A two-tailed $p$ value $<0.05$ was considered indicative of statistical significance.

\section{Abbreviations}

LNM: lymph node metastasis; LLNM: lateral lymph node metastasis; TSH: thyroid-stimulating hormone; ROC: receiver operating characteristic; CLM: central lymph node metastases; PTC: papillary thyroid carcinoma; CND: central neck dissection; LND: lateral neck dissection; CT: computed tomography; MRI: magnetic resonance imaging; ATA: American Thyroid Association; FNAB: fine-needle aspiration biopsy.

\section{Author contributions}

The first authors of this manuscript are Lei JY and Li GP. These two contributed equally to this study and should be co-first authors. Lei JY and Zhu JQ performed the research and wrote the first draft. All authors contributed to the design and interpretation of the study and to further drafts. Zhu JQ is the guarantor.

\section{CONFLICTS OF INTEREST}

No benefits in any form have been received or will be received from a commercial party related directly or indirectly to the subject of this article.

\section{FUNDING}

This study was supported by grants from National Natural Science Foundation (81702646), Sichuan Province Science and Technology Project of China (No.2017SZ0139), Sichuan University for youth fund (2017SCU11016), Health and Family Planning Commission of Sichuan Province (17PJ398), and Postdoctoral Sustentation Fund of Sichuan University (2017SCU12035).

\section{REFERENCES}

1. Haugen BR, Alexander EK, Bible KC, Doherty GM, Mandel SJ, Nikiforov YE, Pacini F, Randolph GW, Sawka AM, Schlumberger M, Schuff KG, Sherman SI, Sosa JA, et al. 2015 American Thyroid Association Management Guidelines for Adult Patients with Thyroid Nodules and Differentiated Thyroid Cancer: The American Thyroid Association Guidelines Task Force on Thyroid Nodules and Differentiated Thyroid Cancer. Thyroid. 2016; 26:1-133.

2. Siegel RL, Miller KD, Jemal A. Cancer statistics, 2016. CA Cancer J Clin. 2016; 66:7-30.

3. Chen W, Zheng R, Baade PD, Zhang S, Zeng H, Bray F, Jemal A, Yu XQ, He J. Cancer statistics in China, 2015. CA Cancer J Clin. 2016; 66:115-132.

4. Kouvaraki MA, Shapiro SE, Fornage BD, Edeiken-Monro BS, Sherman SI, Vassilopoulou-Sellin R, Lee JE, Evans DB. Role of preoperative ultrasonography in the surgical management of patients with thyroid cancer. Surgery. 2003; 134:946-954. discussion 954-945.

5. Lee YM, Sung TY, Kim WB, Chung KW, Yoon JH, Hong SJ. Risk factors for recurrence in patients with papillary thyroid carcinoma undergoing modified radical neck dissection. Br J Surg. 2016; 103:1020-1025.

6. Lan X, Sun W, Zhang H, Dong W, Wang Z, Zhang T. A Meta-analysis of Central Lymph Node Metastasis for Predicting Lateral Involvement in Papillary Thyroid Carcinoma. Otolaryngol Head Neck Surg. 2015; 153:731-738. 
7. Ito $\mathrm{Y}$, Tomoda $\mathrm{C}$, Uruno $\mathrm{T}$, Takamura $\mathrm{Y}$, Miya $\mathrm{A}$, Kobayashi K, Matsuzuka F, Kuma K, Miyauchi A. Ultrasonographically and anatomopathologically detectable node metastases in the lateral compartment as indicators of worse relapse-free survival in patients with papillary thyroid carcinoma. World J Surg. 2005; 29:917-920.

8. Kim E, Park JS, Son KR, Kim JH, Jeon SJ, Na DG. Preoperative diagnosis of cervical metastatic lymph nodes in papillary thyroid carcinoma: comparison of ultrasound, computed tomography, and combined ultrasound with computed tomography. Thyroid. 2008; 18:411-418.

9. Goropoulos A, Karamoshos K, Christodoulou A, Ntitsias T, Paulou K, Samaras A, Xirou P, Efstratiou I. Value of the cervical compartments in the surgical treatment of papillary thyroid carcinoma. World J Surg. 2004; 28:1275-1281.

10. Park JH, Lee YS, Kim BW, Chang HS, Park CS. Skip lateral neck node metastases in papillary thyroid carcinoma. World J Surg. 2012; 36:743-747.

11. Sivanandan R, Soo KC. Pattern of cervical lymph node metastases from papillary carcinoma of the thyroid. Br J Surg. 2001; 88:1241-1244.

12. Roh JL, Park JY, Park CI. Total thyroidectomy plus neck dissection in differentiated papillary thyroid carcinoma patients: pattern of nodal metastasis, morbidity, recurrence, and postoperative levels of serum parathyroid hormone. Ann Surg. 2007; 245:604-610.

13. Roh JL, Kim JM, Park CI. Central cervical nodal metastasis from papillary thyroid microcarcinoma: pattern and factors predictive of nodal metastasis. Ann Surg Oncol. 2008; 15:2482-2486.

14. Zeng RC, Zhang W, Gao EL, Cheng P, Huang GL, Zhang $\mathrm{XH}, \mathrm{Li} \mathrm{Q}$. Number of central lymph node metastasis for predicting lateral lymph node metastasis in papillary thyroid microcarcinoma. Head Neck. 2014; 36:101-106.

15. Zhu Y, Zheng K, Zhang H, Chen L, Xue J, Ding M, Wu K, Wang Z, Kong L, Chen X. The clinicopathologic differences of central lymph node metastasis in predicting lateral lymph node metastasis and prognosis in papillary thyroid cancer associated with or without Hashimoto's thyroiditis. Tumour Biol. 2016; 37:8037-8045.

16. Machens A, Hauptmann S, Dralle H. Lymph node dissection in the lateral neck for completion in central node-positive papillary thyroid cancer. Surgery. 2009; 145:176-181.

17. Xiao GZ, Gao L. Central lymph node metastasis: is it a reliable indicator of lateral node involvement in papillary thyroid carcinoma? World J Surg. 2010; 34:237-241.

18. Lee YS, Lim YS, Lee JC, Wang SG, Kim IJ, Lee BJ. Clinical implication of the number of central lymph node metastasis in papillary thyroid carcinoma: preliminary report. World J Surg. 2010; 34:2558-2563.

19. Ito Y, Tomoda C, Uruno T, Takamura Y, Miya A, Kobayashi K, Matsuzuka F, Kuma K, Miyauchi A. Clinical significance of metastasis to the central compartment from papillary microcarcinoma of the thyroid. World J Surg. 2006; 30:91-99.
20. Giordano D, Valcavi R, Thompson GB, Pedroni C, Renna L, Gradoni P, Barbieri V. Complications of central neck dissection in patients with papillary thyroid carcinoma: results of a study on 1087 patients and review of the literature. Thyroid. 2012; 22:911-917.

21. Caliskan M, Park JH, Jeong JS, Lee CR, Park SK, Kang SW, Jeong JJ, Chung WY, Park CS. Role of prophylactic ipsilateral central compartment lymph node dissection in papillary thyroid microcarcinoma. Endocr J. 2012; 59:305-311.

22. Lee YS, Shin SC, Lim YS, Lee JC, Wang SG, Son SM, Kim IJ, Lee BJ. Tumor location-dependent skip lateral cervical lymph node metastasis in papillary thyroid cancer. Head Neck. 2014; 36:887-891.

23. Chung YS, Kim JY, Bae JS, Song BJ, Kim JS, Jeon HM, Jeong SS, Kim EK, Park WC. Lateral lymph node metastasis in papillary thyroid carcinoma: results of therapeutic lymph node dissection. Thyroid. 2009; 19:241-246.

24. Lim YS, Lee JC, Lee YS, Lee BJ, Wang SG, Son SM, Kim IJ. Lateral cervical lymph node metastases from papillary thyroid carcinoma: predictive factors of nodal metastasis. Surgery. 2011; 150:116-121.

25. Tamimi DM. The association between chronic lymphocytic thyroiditis and thyroid tumors. Int J Surg Pathol. 2002; 10:141-146.

26. Souza SL, Montalli Da Assumpcao LV, Ward LS. Impact of previous thyroid autoimmune diseases on prognosis of patients with well-differentiated thyroid cancer. Thyroid. 2003; 13:491-495.

27. Kim HS, Choi YJ, Yun JS. Features of papillary thyroid microcarcinoma in the presence and absence of lymphocytic thyroiditis. Endocr Pathol. 2010; 21:149-153.

28. Chen Q, Zou XH, Wei T, Huang QS, Sun YH, Zhu JQ. Prediction of ipsilateral and contralateral central lymph node metastasis in unilateral papillary thyroid carcinoma: a retrospective study. Gland surgery. 2015; 4:288-294.

29. Huang SH, O'Sullivan B. Overview of the 8th Edition TNM Classification for Head and Neck Cancer. Current treatment options in oncology. 2017; 18:40.

30. Cooper DS, Doherty GM, Haugen BR, Kloos RT, Lee SL, Mandel SJ, Mazzaferri EL, McIver B, Pacini F, Schlumberger M, Sherman SI, Steward DL, Tuttle RM. Revised American Thyroid Association management guidelines for patients with thyroid nodules and differentiated thyroid cancer. Thyroid. 2009; 19:1167-1214.

31. Xiang D, Xie L, Xu Y, Li Z, Hong Y, Wang P. Papillary thyroid microcarcinomas located at the middle part of the middle third of the thyroid gland correlates with the presence of neck metastasis. Surgery. 2015; 157:526-533.

32. Kim SK, Park I, Woo JW, Lee JH, Choe JH, Kim JH, Kim JS. Predictive Factors for Lymph Node Metastasis in Papillary Thyroid Microcarcinoma. Ann Surg Oncol. 2016; 23:2866-2873. 